\title{
Anabases
}

ANABASES Traditions et réceptions de l'Antiquité

$14 \mid 2011$

Varia

\section{L'imaginaire de la représentation dans Le Théâtre des Grecs de Brumoy (1730)}

\section{Claire Lechevalier}

\section{(2) OpenEdition}

1 Journals

Édition électronique

URL : http://journals.openedition.org/anabases/2162

DOI : 10.4000/anabases.2162

ISSN : 2256-9421

Éditeur

E.R.A.S.M.E.

Édition imprimée

Date de publication : 1 octobre 2011

Pagination : 75-86

ISSN : 1774-4296

\section{Référence électronique}

Claire Lechevalier, "L'imaginaire de la représentation dans Le Théâtre des Grecs de Brumoy (1730) »,

Anabases [En ligne], 14 | 2011, mis en ligne le 01 octobre 2014, consulté le 20 octobre 2019. URL

http://journals.openedition.org/anabases/2162 ; DOI : 10.4000/anabases.2162

\section{(c) Anabases}


Anabases 14 (2011), p. 75-86.

\section{L'imaginaire de la représentation dans Le Théâtre des Grecs de Brumoy (1730)}

Claire Lechevalier

La parution du ThéATRE DES GRECS DE Brumoy, en 1730, répond à un objectif clairement affiché : faire connaître l'ensemble des tragédies et des comédies grecques, injustement oubliées. Il s'agit pour l'auteur de faire renaître le théâtre ancien de ses cendres, de "le rebâtir [...] de ses propres débris ${ }^{1}$ ». Pour autant, il ne saurait être question, ni de traduire la totalité des pièces, ni, qui plus est, d'offrir un texte destiné à la représentation. Qu'elles soient considérées comme trop difficiles à comprendre, trop choquantes au regard de l'esthétique classique, ou bien encore nettement incompatibles avec le " génie " de la langue française, la majorité des pièces resteront donc non traduites, et seront remplacées par des " analyses raisonnées ». On imagine alors bien que, si la traduction, en soi, paraît souvent impossible, l'éventualité de la représentation sur la scène française de 1730 ne soit pas abordée. Il faudra attendre 1844 pour assister à ce que l'on a coutume de considérer comme la première mise en scène française d'une pièce du théâtre antique : l'Antigone de Sophocle au théâtre de l'Odéon.

Pourtant, les premières décennies du XVIII ${ }^{\mathrm{e}}$ siècle voient se développer un véritable débat concernant la possibilité de faire revivre le théâtre antique sur la scène, voire d'en faire un modèle pour une tragédie classique dont l'on commence à redouter l'essoufflement. Si la réflexion peut se développer dans les textes théoriques (on songera par exemple aux Réflexions critiques sur la poésie et la peinture de l'Abbé Dubos, 1719), elle

1 "Discours sur le théâtre des Grecs ", in Le Théatre des Grecs, par le R.P. BrumoY, de la Compagnie de Jésus, à Paris, chez Rollin Père, Jean-Baptiste Coignard et Rollin fils, 1730, t. I, p. xiv. Nous nous attacherons ici à cette édition, et non aux multiples rééditions qui ont été considérablement remaniées. 
donne aussi lieu à des expériences scéniques accomplies, qu'il s'agisse de la création de l'Électre de Longepierre en 1702, des représentations de l'Iphigénie en Tauride restituée par Malézieu pour la duchesse du Maine en 1713, ou des soubresauts de l'élaboration de l'Edipe de Voltaire en $1718^{2}$. À chaque fois, l'on cherche à retrouver la "simplicité " supposée de la tragédie grecque, la force et la grandeur de ses intrigues sans amour, en même temps que l'émotion d'une dramaturgie que l'on imagine volontiers proche de l'enfance de l'art. Pour "légitimer le modèle grec", on fait alors confiance, comme l'a montré Jean-Philippe Grosperrin, à l'« expérience » collective de la représentation, bien davantage qu'aux réflexions théoriques de la poétique.

C'est dans ce contexte, au croisement des perspectives, théoriques et expérimentales, qu'il faut peut-être relire Le Théatre des Grecs, que son auteur lui-même s'attache à présenter comme une "poétique par les faits ${ }^{3}$ ". C'est en effet par l'expérience de la lecture des œuvres elles-mêmes, et non par l'appréciation de leur conformité à la théorie aristotélicienne, que l'on pourra selon lui se faire une idée et construire son jugement concernant le thêâtre grec. Et pour que cette expérience soit plus probante, le lecteur est constamment invité à imaginer la représentation elle-même, voire à se faire, autant que possible, athénien. Ainsi se dessine, ou plutôt s'esquisse ce que l'on se propose d'appeler ici un « imaginaire de la représentation » du théâtre antique, imaginaire trop souvent méconnu, qui trouve pourtant sa place dans ce que pourrait être une archéologie de la représentation du théâtre antique en même temps qu'il informe peutêtre aussi l'histoire de la scène française elle-même. C'est cet imaginaire qu'il s'agira ici de tenter de reconstruire - tout du moins en ce qui concerne la tragédie grecque -, afin d'en analyser les enjeux et les implications, en montrant qu'il s'inscrit non seulement dans la représentation collective que l'on se fait en 1730 du théâtre antique, mais aussi dans la pratique spécifique du théâtre des jésuites, en même temps qu'il porte la trace des remises en cause de l'esthétique théâtrale contemporaine.

L'un des objectifs du Discours sur le théâtre des Grecs, que Brumoy place en tête de son ouvrage, consiste à préparer l'accueil du lecteur contemporain, qui pourrait être tenté, dans la mouvance de la querelle des Anciens et des Modernes, d'aduler en suivant les " autorités » ou bien au contraire de rejeter, comme incompatible avec le monde moderne, un théâtre trop vite jugé. Refusant toute forme de préjugé en même temps que toute comparaison globale avec le théâtre français, Brumoy invite le lecteur à se faire son propre jugement, et à examiner une à une les œuvres comme autant de pièces du dossier, l'expérience de lecteur se substituant alors à la théorie critique. Et

2 Cf. J.-P. GROSPERRIN, « Résurrections princières de la tragédie grecque à la fin du règne de Louis XIV : l'Électre de Longepierre (1702) et l'Iphigénie en Tauride de Malézieu (1713) », Anabases 2 (2005), p. 115-145, et VolTaire, Lettres sur Edipe [1719], dans Euvres complètes, Paris, Garnier Frères, 1877, Théâtre, t. I.

3 Brumor, Le Théâtre des Grecs, t. I, p. xxv. Pour une analyse plus développée du projet de Brumoy, voir C. Lechevalier, L'Invention d'une Origine. Traduire Eschyle en France de Lefranc de Pompignan à Mazon : Le Prométhée enchaîné, Paris, Champion, 2007. 
pour guider ce dernier dans son examen, une méthode est proposée, méthode selon laquelle il est appelé, pour mieux découvrir Alceste par exemple, à faire "un effort d'imagination ", et à se transporter à Athènes "pour voir agir ses acteurs, et [se] prêter à tout le spectacle, sans faire attention à ce qu'[il] li[t] ", bref, à devenir Athénien ${ }^{4}$. Alors seulement les " applaudissements " seront possibles. L'imaginaire de la représentation se comprend donc tout d'abord comme un effort pour appréhender la pièce dans son contexte de production, effort nécessaire au relativisme historique qui doit guider la formation du jugement. Pour éviter une réception trop directement orientée par les habitudes de la tragédie classique ou de la scène contemporaine, Brumoy propose de faire connaître, comme préalable à la lecture des textes eux-mêmes, un ensemble d'informations concernant les cadres et les codes de la représentation antique, informations destinées à permettre au lecteur de se construire mentalement un univers théâtral donné comme historique :

"On a donné beaucoup à la théorie sur les traces d'Aristote et même à la pratique, comme M. l'Abbé d'Aubignac. Il y manquait d'exposer le théâtre ancien dans le point où il faut l'envisager pour le bien connaitre, c'est-à-dire en lui-même par l'exposition des œuvres tragiques et comiques, jointes à la manière dont elles ont été composées, et aux conjonctures des lieux, et des temps qui en sont inséparables. Car c'est sur le rapport de toutes ces choses qu'on peut et qu'on doit décider du prix de ces œuvres, soit en ellesmêmes, soit par égard aux modernes. C'est ici, à proprement parler, une instruction du procès suivant les coutumes du pays grec, choses nécessaires à des juges qu'on ne veut ni surprendre, ni solliciter à prendre parti ${ }^{5}$."

Le Discours sur l'origine de la tragédie aborde donc successivement la question de la " disposition des chœurs" (nombre des participants, mouvements, chants...) ou de l'alternance métrique, autant d'éléments que la traduction en prose ne pourra restituer, et s'attache aussi à décrire l'architecture du théâtre d'Athènes, les décors et les machines, les masques et les costumes utilisés. Brumoy rejoint ainsi un intérêt croissant, dans ces premières décennies du XVIII ${ }^{\mathrm{e}}$ siècle, pour la reconstitution de l'univers dramatique de la Grèce et de Rome. Comme le fait aussi l'Abbé Dubos dans ses Réflexions critiques sur la poésie et la peinture 6 , il puise majoritairement ses connaissances dans les sources textuelles anciennes, en particulier chez Vitruve, qu'il cite ${ }^{7}$, mais aussi dans les récents travaux de l'Académie des inscriptions. Mais là où Boindin, auteur d'un Discours sur la forme et la construction du théatre des anciens, évoque une architecture tripartite ( L'espace compris entre les deux demi-cercles, était la partie destinée aux spectateurs : le carré qui les terminait, celle qui appartenait aux acteurs, et l'intervalle qui restait au

4 Brumoy, Le Théâtre des Grecs, t. I, p. ix.

5 Ibid., p. xxvij-xxviij.

6 Réflexions critiques sur la poésie et la peinture [1719], Paris, Mariette, 1733.

7 Brumoy, Le Théâtre des Grecs, t. I, p. xcvj sq. 
milieu, ce qu'ils appelaient l'orchestre $\left.{ }^{8} »\right)$, s'attachant ensuite à définir plus précisément le rôle de "l'orchestre ", Brumoy simplifie et ne conserve qu'une structure bipartite : "Le demi-cercle contenait les spectateurs rangés par étages les uns au-dessus des autres, et le carré long servait aux acteurs et aux spectacles ${ }^{9}$. " La recherche historique se trouve donc modulée par la volonté de présenter un texte simple. En même temps, la réduction à deux espaces donne à imaginer une scène unique sur laquelle évoluent conjointement acteurs et choreutes, dans une scénographie qui brise toute prise en compte de la séparation spécifique à la tragédie grecque, telle qu'on la représentera à partir de 1844. Cette reconstitution se trouve par ailleurs modulée par la splendeur supposée des spectacles antiques : " Il suffit, pour en juger, de se rappeler que les frais du théâtre et des pièces se faisaient aux dépens de l'état chez les Athéniens, et qu'ils dépensèrent plus pour ces sortes de divertissement que pour plusieurs de leurs guerres ${ }^{10}$. » Ainsi Brumoy est-il conduit, comme le fera plus tard Diderot dans les Entretiens sur le fils naturel, à imaginer une scène immense ( on y voyait des palais, des temples, des places en perspectives et des villes dans l'enfoncement $\left.{ }^{11} »\right)$, lieu de toutes les perspectives, de tous les spectacles et de toute la pompe possible, tels le char de Clytemnestre et son escorte dans Iphigénie en Aulide ${ }^{12}$, ou la chute de Troie dans Les Troyennes ${ }^{13}$. La reconstitution juxtapose souci d'exactitude historique et comparaison sous-jacente avec les scènes françaises, qui, encombrées des banquettes destinées aux spectateurs, sont de plus en plus dénoncées pour leur exiguïté. La recherche historique se mêle alors à la projection d'une scène idéale, l'ensemble se donnant peut-être progressivement à lire comme un modèle de renouvellement pour le théâtre français.

Si les trois Discours inaugurant Le Théâtre des Grecs visent, pour une part, à poser les cadres historiques de la représentation imaginaire, les notes qui accompagnent les traductions des pièces, et les " analyses raisonnées " qui remplacent ces dernières lorsqu'elles sont considérées comme irréalisables, dessinent ensuite des spectacles plus précis. Selon la perspective pédagogique qui est aussi celle du jésuite enseignant au Collège de Louis-le-Grand (1725-1731), auteur de plusieurs tragédies ${ }^{14}$, futur traduc-

8 M. Boindin, Discours sur la forme et la construction du théâtre des anciens, dans Mémoires de l'Académie des inscriptions, t. I, 2 p. 138. Brumoy cite aussi son Mémoire sur les masques, 1712 , t. IV, p. 132.

9 Brumor, Le Théâtre des Grecs, t. I, p. xcv.

10 Ibid., p. xcvj.

11 Ibid.

12 "Quand on fait réflexion à la grandeur des théâtres antiques, on ne doit plus être surpris d'y voir paraître un équipage complet. " (Le Théâtre des Grecs t. I, p. xxxj, note).

13 "Ce concert lugubre est interrompu par la vue des flammes qui paraissent sur les tours, et aux toits des maisons qui restaient encore sur pied dans Troie. Le chœur aperçoit des hommes, la torche à la main, courir ça et là comme des furieux, et porter partout la désolation et l'incendie " ("Analyse des Troyennes", dans Le Théâtre des Grecs, t. II, p. 585).

On citera notamment Isaac, Jonathas, et Le Couronnement de David. 
teur du Discours sur les spectacles de Charles Porée (1733), la puissance du théâtre repose en effet sur la force des images qu'il est capable de produire, suscitant dans un même moment au plus haut point ces deux sens supérieurs que sont la vue et l'ouïe ${ }^{15}$. En l'absence de représentation réelle, le lecteur est invité à se faire une image mentale, comme l'élève des Jésuites doit s'y exercer quotidiennement par la pratique des Exercices spirituels, qui reposent sur une spiritualité appréhendée par la constitution progressive d'images intérieures (lieu, personnages, histoire), faisant le plus large appel à tous les sens. Les " analyses raisonnées " peuvent se comprendre dans cette perspective, qui mêlent passages traduits et récits de l'intrigue, construisant un résumé de l'œuvre où texte original et reconstitution deviennent progressivement indistincts. La pratique, que l'on pourrait considérer comme un héritage de l'interpretatio, dans laquelle traduction, paraphrase et commentaire apparaissaient mêlés, vise à contourner l'écueil de l'intraduisible, constamment réaffirmé par Brumoy, en même temps qu'à substituer à un texte souvent difficile un récit clair et fluide, quitte à des approximations souvent reconnues par l'auteur lui-même ${ }^{16}$. Mais elle permet aussi de faire entendre la voix des personnages en les situant au cœur d'un décor, d'une action, d'une image recréée, partant, de faire revivre de façon concrète le spectacle même du théâtre grec. L'imaginaire apparaît alors avant tout comme un facteur de liberté, en ce que, bien davantage que la pièce théâtrale, le récit laisse la porte ouverte à toutes les représentations quelles qu'elles soient, aussi irréalisables puissent-elles paraître sur une scène française. Le commentaire, intégré aux analyses ou rejeté dans les notes, vient parfois souligner le décalage, et vise souvent à prévenir une éventuelle répulsion. Ainsi souligne-t-il le risque d'invraisemblance à qui voudrait imaginer la métamorphose d'Io en génisse ("Personne ne se persuadera, avec Monsieur Dacier, qu'elle paraisse sur le théâtre en forme de génisse. [...] C'est une imagination trop ridicule pour être fondée. Il suffit que le spectateur soit prévenu que cette fille s'imaginât être métamorphosée ${ }^{17}$ "), excuse, ou tout du moins explique l'horreur par la différence des mentalités ("L'idée seule qu'on avait alors de la fatalité suffisait pour diminuer l'horreur et l'atrocité d'un parricide médité et commis de sang froid $\left.{ }^{18} »\right)$, analyse la rapidité des mouvements ou les ruptures de rythme à l'aune d'une versification spécifique ("Ce sont là des situations que nos vers alexandrins ne peuvent exprimer. Les changements de versification que les Grecs se permettaient, rendent ces sortes de jeux naturels, vifs et incapables d'aucune traduction supportable $\left.{ }^{19} »\right)$. Ainsi l'appareil textuel dans son entier (remarques histori-

15 Voir P.C. Porée, De Theatro, avec la traduction en regard du P. Brumoy, Discours sur les spectacles, É. Flamarion (éd.), Société de Littératures Classiques, Toulouse, 2000, p. 21 sq.

16 "C'est à peu près ainsi, et plus éloquemment encore, que s'exprime la douleur d'Io ", ("Analyse du Prométhée lié ", dans Le Théâtre des Grecs, t. II, p. 158).

17 Ibid.

18 "Réflexions sur l'Électre de Sophocle ", ibid., t. I, p. 197.

19 "Analyse d'Hécube ", ibid., t. II, p. 371. 
ques, dialogues mêlés aux récits, commentaires et notes) vise-t-il à construire progressivement les conditions de possibilité d'une représentation imaginaire, dont Pierre Brumoy s'attache alors, plus précisément, à reconstituer la théâtralité.

Les " analyses raisonnées " décomposent les pièces grecques en cinq actes, selon l'esthétique classique, qui ne reconnaît pas la distinction entre stasima et épisodes. Les passages traduits imposent une langue qui, façonnée par le génie de la langue française, ne peut que trahir la poésie des tragiques, en particulier en ce qui concerne les chœurs $^{20}$. On ne saurait cependant en conclure à une assimilation générale du théâtre antique à la scène française. Brumoy tente en effet d'appréhender la représentation en fonction de ses connaissances historiques, mais aussi des éléments de théâtralité qu'il peut observer dans les textes mêmes. Il souligne à plusieurs reprises le caractère « singulier » des situations que propose le théâtre antique. Les ronflements des Érynnies, ou encore le mélange de joie et de douleur à l'approche des cercueils dans Les Supppliantes d'Euripide, pourraient à juste titre paraître étranges à un lecteur contemporain. L'appréhension par la différence, même difficile à comprendre, permet d'éviter une trop rapide stigmatisation, et d'ouvrir l'intérêt pour une théâtralité singulière que les analyses s'emploient ensuite à explorer. Ainsi se construit peu à peu, entre regard historique, réalité de la scène française et projection d'une scène idéale, une dramaturgie imaginaire de la tragédie grecque, dont l'on peut se demander dans quelle mesure elle n'a pas été fondatrice dans l'histoire des représentations.

Première caractéristique du spectacle antique selon Brumoy, le réalisme qu’il souligne à plusieurs reprises peut paraître au premier abord étonnant. À propos du char de Clytemnestre entrant sur scène dans l'Iphigénie en Aulide, il explique par exemple : "Les Anciens s'appliquaient à rendre l'action théâtrale toute semblable à l'action réelle. Les machines des Anciens étaient plus variées et plus ingénieuses que les nôtres. " Ce sont donc les conditions matérielles, ou tout du moins ce qu'il en connaît (grandeur des théâtres, multiplicité des machines et des décors), qui semblent orienter une représentation dont l'on sait aujourd'hui qu'elle reste bien lointaine de ce que devait être le spectacle fortement conventionnel de la tragédie grecque. Mais la conformité au réel relève bien davantage de cette esthétique de la simplicité, comprise comme expression du naturel, que l'on a coutume, au début du XVIII ${ }^{\mathrm{e}}$ siècle, d'associer au théâtre antique. Évoquant le char de Clytemnestre une nouvelle fois, Brumoy ajoute : "Ce spectacle si naïf ne convenait qu'aux mœurs antiques. Il n'en est pas moins beau pour qui sait priser le naturel ${ }^{21}$. " La simplicité, expression du naturel, partant, de la vérité, oriente donc une théâtralité fondée sur l'observation d'un « objet simple, mais considéré dans

20 "C'est comme si on voulait tourner nos chansonnettes en grec. Un tour en toute langue vaut souvent une pensée, et en est véritablement une. Mais c'est une manne qui fond, un fantôme qui s'évanouit, ou du moins une fleur qui se fane dans une langue étrangère " (Discours sur le théâtre des Grecs, ibid., t. I, p. xxj).

21 Iphigénie en Aulide, ibid., t. I, p. xxxj, note. 
toutes ses situations ${ }^{22}$ ", bien loin de ce que proposent les scènes françaises appelées à satisfaire un public friand de changements et d'artifices. Ainsi l'auteur du Théâtre des Grecs souligne-t-il la lenteur et la plénitude du récit du " courrier » dans Les Perses :

« La manière dont le récit interrompu du courrier se mêle aux gémissements du Chœur est inexprimable. C'est la nature elle-même. En effet, dans l'usage ordinaire, il est assez peu naturel qu'un homme fasse de suite une longue narration d'un malheur domestique, sans qu'on l'interrompe par des cris, des réflexions, des questions : et cependant c'est ce qu'on voit arriver la plupart du temps sur le théâtre par la nécessité d'être court, et de frapper les spectateurs. Mais Eschyle atteint ici l'un et l'autre but en suivant les mouvements naturels, et en imitant parfaitement ce qui se passe tous les jours. Son récit augmente et croît à proportion des gémissements de ceux qui l'écoutent. Ces gémissements d'ailleurs sont si vifs et si beaux que c'est faire tort à Eschyle de les laisser passer, et plus encore de les exprimer, tant il est difficile d'attraper ce naïf précieux qui règne dans toute l'antiquité grecque ! ce sont les images les plus fortes que la douleur emploie, des images de vaisseaux brisés, de corps flottants, d'épouses abandonnées, de veuves, d'orphelins, etc. choses qui nous paraîtraient admirables, si nous étions dans la même situation que les spectateurs $\operatorname{grecs}^{23}$."

Cette même naïveté justifie aussi, à l'opposé, la rapidité ou la vivacité de certains jeux de scène. Dans Hécube, Polymestor, bouleversé à la découverte de ses enfants massacrés, " court ça et là, sans tenir de route assurée. Il poursuit en vain les femmes qui l'ont assassiné. Il appelle à son secours les Grecs, les Atrides, et toute l'armée ${ }^{24}$... ». De tels mouvements pourraient paraître incongrus au public français, habitué aux postures codées de la déclamation, mais ils se trouvent ici valorisés comme " naturels, vifs ", quoique "incapables d'aucune traduction supportable ». Ainsi se construit une représentation fondée sur l'idée d'un théâtre vu comme expression de l'enfance de l'art, d'une parole entendue comme imitation du réel, bien loin de la poésie complexe que l'on observe aujourd'hui chez les tragiques grecs. En même temps, l'éloge du naturel permet de réhabiliter, à travers la valorisation de l'émotion et de ses moyens d'expression, un théâtre encore peu traduit en France, et d'introduire un point de comparaison, voire un outil de critique pour la scène contemporaine.

La naïveté n'exclut cependant pas le spectaculaire. Brumoy affirme sans cesse la dimension visuelle de la tragédie grecque, réhabilitant par là-même la valeur de l'opsis, que la tradition aristotélicienne avait largement altérée ${ }^{25}$. C'est tout d'abord la présence de l'action, contre le discours, qui se trouve réaffirmée. À propos des

22 Discours sur le parallèle des théâtres, ibid., t. I, p. cxlij.

23 "Analyse des Perses", ibid., t. II, p. 175.

24 "Analyse d'Hécube», ibid., t. II, p. 371.

25 Aristote, Poétique, Roselyne Dupont-Roc et Jean Lallot (éd.), Paris, Seuil, 1980, chapitre 14 . 
Suppliantes d'Euripide : «Le spectacle et le jeu de l'action faisaient une des principales parties des tragédies anciennes ${ }^{26}$." L'observation se comprend une fois encore en lien avec l'évolution de la scène française, à laquelle le théâtre grec est toujours comparé : "C'est plus l'action et le spectacle que les paroles, et plus la passion et le sentiment que le discours ; au lieu que les Français ont souvent donné dans le discours et les paroles, pour suppléer au spectacle et à la passion ${ }^{27}$. " Elle rejoint une revendication plus générale des contemporains, qui, tels La Motte ou Voltaire, regrettent de plus en plus le manque d'action des tragédies françaises et en appellent au développement du spec$\operatorname{tacle}^{28}$. Elle suscite une analyse de chaque situation scénique, pour laquelle Brumoy s'attache à reconstruire les différents plans de l'espace, la position des personnages, ou l'utilisation des machines. Au moment où Iphigénie affirme à sa mère, Clytemnestre, sa volonté de marcher au sacrifice, à la fin de l'Iphigénie en Aulide (v. 1466), Brumoy ajoute en note à sa traduction :

"Il est croyable que Clytemnestre tombe évanouie, et qu'on l'enlève dans le palais, tandis qu'Iphigénie exhorte le chœur à chanter des hymnes en l'honneur de Diane. On devait voir au fond du théâtre une partie de l'appareil du sacrifice : le chœur semble en être le témoin oculaire ${ }^{29}$."

Dans le texte grec, Iphigénie se tourne certes vers le chœur, avec lequel elle entre en commos, mais c'est pour lui ordonner, devant sa mère probablement toujours présente $^{30}$, la préparation du sacrifice, qui se déroulera hors scène. L'ensemble se passe à Aulis, dans le camp grec, devant les tentes. Brumoy reconstitue ici la scène dans la perspective réaliste qui est la sienne, ou tout du moins selon l'idée, explicitée par ailleurs, que les événements se passaient "réellement » comme le spectateur le voyait "sous ses yeux ${ }^{31}$ ". En fonction du matériau énonciatif et thématique du texte, il reconstruit trois actions (mouvements de Clytemnestre, adresse d'Iphigénie au chœur, préparation du sacrifice), qui s'organisent en trois plans (" le palais ", la scène elle-

26 "Analyse d'Hécube", dans Le Théâtre des Grecs, t. II, p. 536.

27 Discours sur le parallèle des théâtres, ibid., t. I, p. cxliv.

28 "La plupart de nos pièces ne sont que des dialogues et des récits; et ce qu'il y a de plus surprenant, c'est que l'action même qui a frappé l'auteur et l'a déterminé à choisir son sujet, se passe presque toujours derrière le théâtre " (A. Houdar DE La MotTE, Second Discours à l'occasion de la tragédie de Romulus, dans CEuvres de théatre. Avec plusieurs discours sur la tragédie, Paris, Dupuis, 1730, t. I, p. 118) ; les tragédies françaises "sont plutôt des conversations qu'elles ne sont la représentation d'un événement " (VOLTAIRE, Discours sur la tragédie, en tête de Brutus, dans Euvres complètes, Voltaire Foundation, Oxford, 1998, t. 5, p. 164).

29 "Note à la traduction de l'Iphigénie en Aulide", dans Le Théâtre des Grecs, t. I, p. lxxxij.

30 Les vers 1487-1490, à travers lesquels Iphigénie s'adresse à sa mère, ne supposent pas nécessairement la présence de cette dernière, mais rien n'indique non plus sa sortie de scène. Dans le texte que traduit Brumoy, c'est le chœur qui prononce ces vers.

31 "Réflexions sur l'Iphigénie en Tauride», dans Le Théâtre des Grecs, t. II, p. 76. 
même, "le fond du théâtre "), selon une conception de l'espace orientée, on l'a vu, par l'idée du gigantisme du lieu et les possibilités supposées de la perspective. L'ensemble se superpose en une image unique, dans laquelle les trois actions sont présentées comme simultanées ("tandis que ») dans une présentation qui structure les lignes et guide le regard imaginaire du lecteur (le chœur est " témoin oculaire "). La juxtaposition des différents plans - qui ignore, on l'a vu, la bi-partition orchestra-logeion telle qu'on la reconstruira ensuite dans l'histoire des représentations -, ajoutée à la co-présence des individus et des groupes, engendre une composition organisée autour d'acteurs et de spectateurs internes, dans un ensemble que l'on peut considérer selon l'" esthétique du tableau " dont Pierre Frantz a montré comment elle se mettait en place dans les premières décennies du XVIII ${ }^{\mathrm{e}}$ siècle ${ }^{32}$.

Le terme apparaît en effet à plusieurs reprises dans les différentes réflexions et analyses, sans pour autant pouvoir être confondu avec le procédé classique de l'hypotypose. La technique picturale vient à l'aide de l'auteur pour mieux appréhender les moyens de la représentation, dont il s'agit aussi d'analyser les différentes composantes. Elle apparaît comme un outil, destiné à rendre explicite la dimension concrète, matérielle, voire technique de la scène. Elle devient même un principe explicite lorsque Brumoy analyse l'ensemble d'Edipe-Roi, comme une succession de " tableaux ", " dignes du pinceau de Raphaël ${ }^{33}$ ». La vue est en effet tellement parlante qu'elle pourrait se passer de mots :

"Voilà un spectacle parlant, et un tableau si bien ordonné, que la seule attitude du sacrificateur et d'Edipe déclarerait sans autres paroles, que l'un expose les maux dont la ville est affligée, et que l'autre attendri à cette vue témoigne son impatience $[\ldots]^{34}$.»

L'on aurait cependant tort de réduire ces tableaux à des images arrêtées, comme l'opéra en produit à l'époque ; il faut peut-être aussi les considérer comme des précurseurs de ces moments de spectacle autonomes que théorisera plus tard Diderot. La tragédie grecque est en effet donnée à voir comme un spectacle composé de séquences successives, au sein duquel l'action l'emporte largement sur les paroles, tant par la domination scénique ("C'est là, selon les apparences, tout le troisième acte, qui consiste, on le voit, beaucoup plus en spectacle et en action qu'en paroles ${ }^{35}$ "), qu'en efficacité dramaturgique ("spectacle plus frappant que les vers ${ }^{36}$ "). Ainsi l'ensemble ne pourrait-il s'appréhender par une lecture qui n'ait pas recours à l'imaginaire de la représentation ("Tout cela est plus proche du spectacle que de la lecture ${ }^{37}$ »). Dans

32 P. Frantz, L'Esthétique du tableau dans le théatre du XVIII eiècle, Paris, PUF, 1998.

33 "Réflexions sur l'Edipe", dans Le Théâtre des Grecs, t. I, p. 88.

34 Ibid., p. 89.

35 "Analyse des Perses", ibid., t. II, p. 179.

36 "Analyse des Euménides", ibid., t. II, p. 207.

37 «Analyse des Suppliantes d'Euripide », ibid., t. II, p. 546. 
cette perspective, le dialogue des personnages, et plus encore le chant du chœur, rarement traduit, se trouvent réduits à une thématique, souvent non développée. La pratique du résumé est pour une part imposée par les contraintes de l'« analyse raisonnée ", mais les choix qu'opère Brumoy montrent que c'est avant tout l'acte de parole, sa fonction pragmatique qui importe, bien davantage que la signification purement littérale, lexicale ou grammaticale. Le procédé domine en particulier lorsqu'il s'agit des tragédies d'Eschyle - dont la " naïveté ", partant l'émotion jaillissante, voire non mise en forme, est supposée l'emporter - et peut aussi trouver son explication dans la difficulté de compréhension littérale que posent en particulier les chants du chœur. Ainsi l'« hymne » des Érynnies autour d'Oreste se trouve-t-il résumé en ces termes :

" En signe de réjouissance de l'avoir retrouvé, elles se mettent à chanter autour de lui une ode magique et infernale. Cette espèce d'hymne est pleine du feu d'Eschyle, et inspire je ne sais quelle horreur. Plusieurs des couplets finissent par un refrain qui montre que ce chant est un chant de furies, un chant qui lie les hommes coupables et qui les fait sécher de frayeur. Tout ce qu'elles disent tend à faire voir qu'elles sont les vengeresses de la justice et les bourreaux des criminels ${ }^{38}$."

Les quelque cent vers du chant du chœur se trouvent donc résumés autour de quelques termes directement traduits (« hymne », (v. 331), « un chant qui lie» (v. 331), " sécher de frayeur " (v.332)...), choisis en fonction de leur valeur pragmatique, voire métathéâtrale, l'ensemble oscillant entre résumé, commentaire et indications de mise en scène. Le procédé apparaît aussi concernant les tragédies d'Euripide (le commos des Suppliantes, par exemple, au moment où sont portés sur la scène les cadavres des chefs (troisième épisode), est présenté comme un "mélange de joie et de douleur, de triomphe et de deuil »). De façon plus générale, les analyses soulignent la présence récurrente de la lamentation sous toutes ses formes (" concert lugubre ", " gémissements ", "plaintes ", "pleurs "), comme constitutive du spectacle de la tragédie grecque. Ainsi se construit progressivement une représentation de cette dernière comme " cérémonie ", à laquelle la dimension rituelle peut parfois donner « un air tout à fait magique et théâ$\operatorname{tral}^{39}$ ». Brumoy semble donc revenir à ce qu'il considère comme une source vive, voire un archétype dramaturgique, et retrouver le secret d'un théâtre religieux, d'un théâtre qui jouerait de ses spécificités propres : la puissance du spectacle et de l'émotion qu'il produit plutôt que le recours au discours, la suggestion magique du chant plutôt que la raison des mots, la terreur religieuse plutôt que la pusillanime galanterie.

Il serait peut-être tentant d'assimiler l'ensemble des caractéristiques de la représentation ainsi reconstituée (profondeur des espaces, spectaculaire, simplification du dialogue, cérémonie) à celles que l'on trouve alors dans la mise en scène de l'opéra, et dont Houdar de la Motte montre par ailleurs qu'elles devraient inspirer la scène tragi-

38 "Analyse des Euménides », ibid., t. II, p. 210.

39 "Analyse des Perses», ibid., t. II, p. 179. 
que française ("L'opéra, malgré ses défauts, a cet avantage sur la tragédie qu’il offre aux yeux bien des actions qu'elle n'ose que raconter $\left.{ }^{40} »\right)$. Brumoy s'inscrirait ainsi dans une longue tradition qui vise, depuis l'Alceste de Quinault, à faire de la tragédie lyrique le genre le plus apte à offrir une reconstitution scénique de la tragédie grecque. On remarquera qu'au contraire, l'auteur du Théâtre des Grecs s'attache à séparer les deux genres, tout en soulignant que certaines pièces, telles les Bacchantes, transcendent la distinction ${ }^{41}$. Celle-ci repose avant tout sur la prise en compte des dimensions morale et religieuse de la tragédie grecque, rendues particulièrement sensibles par une dramaturgie singulière. Le spectaculaire, la puissance de l'émotion, la dimension cérémonielle, ne se comprennent en effet que liés à la vertu qu'ils exaltent, bien loin de l'immoralité affichée, selon Brumoy, à l'opéra. Imaginer la représentation, c'est donc non seulement donner à voir et à entendre, mais aussi tenter de retrouver, par l'analyse, les modalités d'une efficacité perdue, celle d'un théâtre considéré comme école de morale et de vertu.

Entre affirmation de la vérité historique et esthétique théâtrale des jésuites, assimilation à la tragédie française et projection d'une scène idéale, la reconstitution que propose Brumoy apparaît cependant profondément guidée par la curiosité, comme par la volonté de faire renaître une dramaturgie singulière. Ce que propose ici ce dernier, lorsqu'il ne traduit pas mais résume, c'est l'idée d'un spectacle originel au sein duquel la place du texte doit être fortement réévaluée, et les dialogues resitués comme un élément parmi d'autres. C'est l'idée d'un théâtre dont le texte peut être parfois - en particulier lorsqu'il s'agit du chœur - appréhendé comme une suite d'images ou d'effets rhétoriques destinés à faire naître l'émotion, voire comme une suite d'indications pour la mise en scène. On voit alors se construire une dramaturgie imaginaire, qui mêle dialogues et performance, dans un triomphe du spectaculaire dont l'ensemble du XVIII ${ }^{\mathrm{e}}$ siècle, et au premier chef, les remarques de Diderot sur la tragédie grecque, portent nettement la trace ${ }^{42}$. Par delà la réédition de 1785-1789 et l'orientation plus littérale et historique apportée par les traductions et les notes de La Porte du Theil, de Rochefort ou de Prévost, la force des images suscitées en 1730 subsistera encore au début du XIX siècle, comme en témoigne ce «sujet de peinture » relevé par Ingres dans ses cahiers, et largement inspiré par les mots mêmes de l'analyse des Choéphores de Brumoy:

40 A. Houdar de La Motte, Second Discours à l'occasion de la tragédie de Romulus, op. cit., p. 118.

41 À propos de l'impiété de Penthée, il explique : "Cela me ferait presque penser que le caractère de cette tragédie est celui d'un véritable opéra, et qu'il y en a eu apparemment plus d'un modèle dans l'Antiquité. [...] Cette antiquité de l'opéra ne le rendra pas plus innocent à nos yeux, surtout tant que le vice paré des plus brillantes couleurs y triomphera impunément de la vertu. " (Le Théâtre des Grecs, t. II, p. 625).

42 Pour une analyse plus précise de l'influence de Brumoy sur Diderot, voir C. LeCHEVALIER, L'Invention d'une Origine, op. cit., p. 83-88. 
"Oreste, les corps morts d'Égisthe et Clytemnestre, Les Euménides, Électre, le peuple: Oreste, après avoir tué Clytemnestre, fait ouvrir les portes du palais et montre de loin au peuple les corps morts d'Égisthe et de Clytemnestre. Oreste à l'instant croit voir les Euménides et les serpents siffler sur leurs têtes ${ }^{43}$."

Claire LeCHEVALIER

Université de Caen

14, rue du Port

F-91450 Soisy-sur-Seine

claire.lechevalier@free.fr

43 Les mots en italiques sont ceux qui ont été ajoutés par Ingres. Les autres trouvent leur renvoi dans Brumoy. L'ensemble est cité par N. SCHLENOFF, dans Ingres, cahiers littéraires inédits, Paris, PUF, 1956, p. 47. 